\title{
Expressions of Growth Factors in Autologous Derived Platelet-Rich Plasma and Platelet-Poor Plasma; Implication for Tissue Reparation and Wound Healing
}

\author{
Akingboye $\mathrm{AA}^{1,2,4}$, Kyriakides $\mathrm{C}^{\mathbf{1}}$ and Tucker $\mathrm{AT}^{3}$ \\ ${ }^{1}$ Department of Vascular Surgery, London \\ ${ }^{2}$ Centre for Cutaneous Research, Institute of cell and molecular science, London \\ ${ }^{3}$ Barts Healthcare NHS Trust Vascular \& Microvascular Unit, London \\ ${ }^{4}$ Department of General Surgery, London
}

*Corresponding author: Akinfemi A Akingboye, Clinical Research Fellow/ Specialist Registrar, Department of General Surgery,

Colchester Hospital University Foundation Trust, Turner Road, Colchester, CO4 5JL, London

\begin{abstract}
ARTICLE INFO
Received: 幽 May 30, 2019

Published: 蔧 June 07, 2019

Citation: Akingboye AA, Kyriakides C, Tucker AT. Expressions of Growth Factors in Autologous Derived Platelet-Rich Plasma and Platelet-Poor Plasma; Implication for Tissue Reparation and Wound Healing. Biomed J Sci \& Tech Res 18(4)2019. BJSTR. MS.ID.003191.
\end{abstract}

Keywords: Platelet-Rich Plasma; Platelet-Poor Plasma; Growth Factors, Wound Healing

Abbreviations: EGF: Epidermal Growth Factor; FGF-2: Fibroblast Growth Factor; PDGF-AA: Platelet Derived Growth Factor; VEGF: Vascular Endothelia Growth Factor; TGF beta-1: Transforming Growth Factor; IGF: Insulin-like Growth Factor; SCD62P: P-Selectin- Platelet Surface Receptor; PRP: Platelet Rich Plasma; PPP: Platelet Poor Plasma; APC : Autologous Platelet Concentration; GFs: Growth Factors; EDTA: EthyleneDiamine Tetra- Acetic Acid
ABSTRACT

Objectives: The growth factors (GFs) released from platelet-rich plasma (PRP) is proposed to modulate vascular reactivity and the inflammatory process. other GFs implicated in the wound healing cascade includes Platelet derived growth factor (PDGF), transforming growth factor -beta (TGF-beta), insulin-like growth factor (IGF)fibroblast growth factor(FGF) and vascular endothelia growth factor (VEGF) and many others. This study attempts to quantitatively evaluate the level of expressions of these GFs derived from platelet-rich plasma from healthy volunteers and diabetic patients

Method: Samples from 14 healthy donors and 6 diabetic patients were analyzed. Platelet counts from the diabetic patients' whole blood and platelet rich plasma (PRP) were analyzed. GFs levels were measured from autologous derived PRP and Plateletpoor plasma (PPP) using ELISA and multiplex immunoassay (Luminex system).

Results: Predictably we found a 6-fold increase in platelet counts from PRP in both groups compared to the whole blood. GF expression in diabetics did not correlate with the platelet count or with the PRP on the regression analysis ( $\mathrm{r}(\mathrm{p})->0.2<=0.9$.). GFs expressions were similar in healthy donor and diabetic patient. GF level were significantly higher for PRP compared with the PPP in both groups $(\mathrm{P}<0.001)$ for PDGFAA, EGF, VEGF, TGF-beta and P-Sel (P-selectin) while there was a trend to lower levels for FGF-2 and IGF $(\mathrm{P}>0.05)$ in both diabetic and healthy volunteer.

Conclusion: Sequestration and concentration of Platelet rich plasma from whole blood with expression of varying growth factors that have been implicated in wound healing was possible with the use of bed side point of care. Furthermore, we demonstrated that activating Platelet poor plasma also released similar GFs as PRP but at lesser level of expression. We believe that this process may enhance delivery of GFs directly to wounds, thereby enhancing the normal physiological wound healing and tissue reparation processes.

\section{Introduction}

Growth factors (GFs) released from activated platelets initiate and modulate healing in both soft and hard tissues [1-5]. A recent therapeutic strategy to promote wound healing is through preparing autologous platelet concentrate (APC) suspended in plasma, known as platelet-rich plasma [6,7]. Platelet-rich plasma (PRP) acts as a storage vehicles for GFs, of such are PDGF [AA,AB and 
BB], TGF-beta 1 \& 2, FGF- acid and basic, EGF, IGF and VEG [1,2,8,9] These GFs have been identified as a central player in the wound healing cascade and are known to influence the process of tissue regeneration [1]. Several publications have demonstrated that PRP has a great potential in the field of degenerative medicine and tissue reparation process $[3,10]$. However, despite the popularity of the use PRP, this novel technique is still plagued with several flaws of which includes inconsistence in the method of preparations and non- reproducibility of the GF expression when attempts are made to quantify their level of expression.

Some authors have alluded that the inconsistence and the difficulty encounter in ascertaining the quality of PRP preparation at a standardized pattern could be as a result of the followings; poor handling technique, the use of animal-derived thrombin for clotting, and fundamental individual idiosyncrasy [6]. To overcome these drawbacks, Anitua et al. developed plasma rich in growth factors (PRGF) by modifying the procedure of PRP preparation [7] has simplified the preparation protocol and replaced the animalderived thrombin with calcium for clotting. In addition, the Pointof-care devices system has helped to standardize the process of PRP preparation and production, which makes it useable at the bedside. This process involves fractionating the whole blood into PRP, platelet -poor plasma (PPP), PRP and red blood with a buffy layer of white cell coat [11], this is then activated by autologous derived thrombin to create a viscous gel known as PRP. Choukroun et al., demonstrated that the variability in the quality of the PRP gel is a function of the sequestration process and the speed of centrifugation, which he described as advanced plasma rich fibrin (soft fibrin clot) and the concentrated growth factor (stiffer fibrin clot) [12]. Therefore, it has been anticipated that the difference in mechanical characteristics may produce a difference in the growth factors expression [12]. However, it is unknown whether there is variability in the pattern expression of GFs from autologous derived PRP or PPP in diabetic patients and healthy volunteers. The purpose of this study was to compare the levels of growth factors released from PRP/PPP from diabetic patients with the chronic ulcers with healthy donors.

\section{Material and Method}

\section{Study Design}

Six male diabetic patients with chronic foot ulcers, age ranging from 45- 68 years as well as fourteen healthy volunteers ( 5 female and 9 male), age range of 18-40 years were recruited. Research Ethics approval was obtained from the East and City of London Research Ethic committee and informed consent was obtained from all volunteers and patients. In addition, the study protocol conformed to the ethical guidelines of the 1975 Declaration of Helsinki as reflected in approval by the institution's human research review committee. Platelet count from the whole blood and PRP in diabetic patients was analyzed but the platelet count was not measured in healthy donors. The rationale for measuring the Platelet count in the diabetic's patient with other medical condition was to ascertain the platelet count was within normal range $(100,000-300,000 / \mu \mathrm{l})$, which was assumed will be appropriate to generate good quality PRP. In addition, this was used to validate the concentrating capacity of the point-of-care device used in this study (Angle Sorin). GFs level from PRP and PPP in both diabetic patients and healthy donors were measured. These was analyzed using the traditional ELISA technique for P-selectin and IGF, whilst the remaining GFs were measured using multiplex fluorescent immunoassay technique (MFI). MFI has a few advantages over ELISA in that it has increased sensitivity, specificity and better reproducibility. The GFs measured with ELISA were not available on the commercial multiplex kit. However, in a study conducted by research animal diagnostic laboratory (RADIL), they showed a correlation of greater than $99.5 \%$ between MFI ELISA.

\section{Preparation of Platelet-Rich Plasma}

The PRP was routinely processed in a sterile environment. Six-millimeter anticoagulant citrate dextrose -A (ACD-A) solution was drawn into 60 -cc syringe, followed by 54 cubic centimeters of whole blood drawn from a large antecubital vein, the acid dextrose serves to preserve the integrity of the platelet membrane. The blood was gently agitated by mixing the anticoagulant with the blood. The $60 \mathrm{ml}$ of blood was injected into the point of care centrifuge system (Angle, Sorin Group, Mirandola, Italy) and centrifuged for 25 minutes (first hard spin) at 5200rpm, followed by a soft spin at $3200 \mathrm{rpm}$ for another 45 minutes. The whole blood is sequestered into a semi-automatic controlled operation mode by centrifugation, through the whole blood separation processing system (Angel; Sorin), which separates the blood into PRP which contains platelet concentrate and leukocyte, PPP and erythrocytes. The desired product (PRP) is usually $10 \%$ of the starting volume of the whole.

\section{PRP and PPP Activation}

To initiate the release of the GFs from the PRP, the platelets must be activated, using the potent platelet activator thrombin. Human thrombin was generated from a commercial thermogenesis which consists of [ $66 \%$ volume for volume of Ethyl Alcohol, U.SP.25Mm Calcium Chloride U.S.P], stored at between $15-30^{\circ} \mathrm{C}$, as a kit called "Activat" [Sorin Group, Mirandola Modena, Italy 41037]. Thrombin was generated after $12 \mathrm{ml}$ of PPP was mixed with the "Activat" beaded material in a pressurized syringe for about 20-25 minutes and the thrombin was squeezed out of the syringe, which generates a total of $4-6 \mathrm{ml}$. Autologous thrombin is preferred in the United Kingdom to pre-prepared bovine thrombin, which has been implicated in the development of antibodies to clotting factor Va, $\mathrm{XI}$ and thrombin. The harvested PRP was combined with thrombin for platelet activation to produce the gelatinous material in ratio of 1:10 [volume/volume]. Similarly, the PPP was activated using the thrombin in the same ration. Following activation, the formed gelatinous material was left in a $15 \mathrm{ml}$ falcon tube overnight at $4^{\circ} \mathrm{C}$, this allows for maximum clot retraction. The supernatant from the gelatinous coagulum was removed after centrifuging for 10 minutes at $3000 \mathrm{rmp}$ and is stored at $-80^{\circ} \mathrm{C}$, until GFs was ready for analysis. 
The supernatant was much easier to handle when performing the ELISA as compared with the gelatinous coagulum.

\section{Hematology Analysis of Whole Blood and Platelet-Rich Plasma}

Blood samples from diabetic patients $(5 \mathrm{ml}$ of whole blood and 3-5 $\mathrm{ml}$ of inactivated PRP) were retained in EDTA bottles. Each sample was counted in triplicate and then average (mean +/-SD). Complete blood count was carried out with the Beckman Coulter LH750 analyzer. This step was repeated for the platelet-rich samples.

\section{Quantification of Growth factors in Platelet-Rich Plasma and Platelet-Poor Plasma}

The supernatant (post activation) obtained from PRP and PPP that could thaw at room temperature. The MILLIPLEX is based on the luminex Xmap technology, which performs immunoassays on the surface of fluorescent-coded beads known as microspheres [Millipore Corporation 290 Concord rd. Billerica]. IGF and P-Selectin ELISA kits were measured by ELISA (R\&D Minneapolis Minn) according to manufacturer's instructions. Briefly, standards of known concentration and PRP/PPP samples were added to a microwell plate with an antibody against each factor. Any growth factor present was bound by the immobilized receptor. Afterwards, any unbound substances were rinsed away, an enzyme-linked polyclonal antibody specific for each growth factor was added to the wells. After a second wash, a substrate solution was added, and the color developed in proportion to the amount of bound growth factor in the first step. The colour development stops once all the substrate have been bound, this was followed by measuring the colour intensity. On the other hand, concentration of TGF-beta1, EGF, VEGF, FGF-2, PDGF-AA and TGH-beta1 were measured with the MILLIPLEX ${ }^{\text {TM }}$ MAP kit. Samples were initially diluted 1:10 in assay buffer and retested at higher dilution if the median fluorescent intensity (MFI) was higher than the value of the top standard. Samples diluted 1:100 for these assays in the kit diluents. All samples were tested in duplicate. Manufacturer's controls were running and found to fall in the range indicated for the kit.

\section{Statistical Analysis}

Statistical analysis was carried out using a linear model based on log transformed values of the growth factors. PRP and PPP groups for each of the diabetic and healthy groups separately. In addition, the mean difference, 95\% confidence interval and the n-fold increase of PRP to PPP was derived. Relationships between platelet count and growth factors was investigated using a linear regression model. Correlations between growth factors and PRP count and GFs and platelet count were assessed by Pearson's correlations. Normality checks and model checks were also carried out and no major violations of statistical assumptions were noted All analysis was carried out at the 5\% (2 -tail) level of significance.

\section{Results}

\section{Hematology Analysis of Platelet-Rich Plasma}

Complete blood count analysis was performed on the whole blood and platelet-rich plasma samples from the six diabetic patients. The platelet separation system increased the platelet count on average from $334,400 \pm 117,000$ platelet/ $\mu \mathrm{L}$ to $1,995,000$ $\pm 805,000$ platelet $/ \mu \mathrm{L}$. The Platelet baseline value ranged from 130,000 to 500,000 platelet/ $\mu \mathrm{L}$. The platelet separating system resulted in an average 6-fold increase in platelet concentration. The platelet rich plasma group was significantly higher in platelet number than the baseline whole group, with a value $\mathrm{p}>0.0005$.

\section{Quantification of Growth Factors}

The quantification of the GFs from the PRP was determined in both the normal control and the diabetic patients. The expression of the GFs in both groups appeared similar. Conversely, when the expression of GFs from PRP were compared with the PPP; TGFbeta1, EGF, PDGF-AA and VEGF were significantly greater in diabetic patient and the healthy donors (Table 1a).

Table 1a: The expressions of growth factors were measured in duplicate for the 6 diabetic patients.

\begin{tabular}{|c|c|c|c|c|c|}
\hline $\begin{array}{c}\text { Growth factors in } \\
\text { Diabetic }\end{array}$ & Comparison increase & n-fold & $\begin{array}{c}\text { Lower 95\% } \\
\text { (Confidence interval) }\end{array}$ & $\begin{array}{c}\text { Upper 95\% } \\
\text { (Confidence interval) }\end{array}$ & P- Values \\
\hline EGF & PRP vs PPP & 4.5698 & 2.5796 & 8.096 & 1.617 \\
\hline FGF-2 & PRP vs PPP & 1.0414 & 0.6707 & 39.1449 & 1.8853 \\
\hline PDGF-AA & PRP vs PPP & 68.6203 & 68.6203 & 11.4127 & $<0.0001$ \\
\hline VEGF & PRP vs PPP & 4.2307 & 4.2307 & 1.0285 & $<0.00015$ \\
\hline TGFb1 & PRP vs PPP & 27.3338 & 27.3338 & 1.0983 & 2.2172 \\
\hline IGF & PRP vs PPP & 1.0983 & 2.7370 & $<.0078$ \\
\hline SCD62P & PRP vs PPP & 2.7370 & $<.0001$ \\
\hline
\end{tabular}

\section{Comparison of Growth Factor Levels in Diabetic Patients (PRP vs PPP)}

In this study PPP was used as the benchmark in which the $\mathrm{n}$-folds increase for the GFs from the PRP were estimated. The mean content $( \pm$ SD) of PRP versus PPP in EGF was $234.9 \pm 193.8 \mathrm{pg} / \mathrm{ml}$ : $31.9 \mathrm{pg} / \mathrm{ml}$ with 5 fold increase, FGF-2; $49.2 \pm 33.64 \mathrm{pg} / \mathrm{ml}: 49.2 \pm$ $42.21 \mathrm{pg} / \mathrm{ml}$ with no increase, PDGF-AA; $104.8 \pm 101 \mathrm{ng} / \mathrm{ml}: 1.125 \pm$ $0.674 \mathrm{ng} / \mathrm{ml}$ had 68 fold increase; VEGF; $0.888 \pm 0.97 \mathrm{ng} / \mathrm{ml}: 0.167 \pm$ 
$0.180 \mathrm{ng} / \mathrm{ml}$ with a 4 fold increase, TGF-beta1; $28.384 \pm 14.63 \mathrm{ng} / \mathrm{ml}$ with a 27 fold increase. No significant was found in IGF in this study, IGF in PRP was $115.1 \pm 32.54 \mathrm{pg} / \mathrm{ml}: 106.1 \pm 102.2 \mathrm{pg} / \mathrm{ml}$ in PPP. The level of platelet activation was estimated by the concentration of the P-selectin, there was two-fold increase in the value of the P-Selectin in PRP $(161.5 \pm 113.6 \mathrm{pg} / \mathrm{ml})$ compared to $\mathrm{PPP}(65.2 \pm 17.67$ $\mathrm{pg} / \mathrm{ml}$ ), which is statistically significant $<0.0001$ (Table $1 \mathrm{~b}$ ). GF expression in diabetics did not correlate with the platelet count nor with the PRP on the Pearson's correlation coefficients analysis (rp) $(r(p)->0.2 \leq 0.9)$. Relationships between platelet count and growth factors were investigated using a linear regression model, this was only performed for the four most significantly expressed GFs and the $\mathrm{p}$ value results are as follows: PDGF-AA;0.7157, VEGF;0.6616, TGF-1;0.3740, EGF;0.6499. None of the correlations were significant, and the correlations appear to be quite weak (maybe because of the sample size). A scatter plot of these GFs is shown in Figure $1 \mathrm{a}-1 \mathrm{~d}$ with a linear regression line superimposed.Plots with Linear Regression Line superimposed for the 4 most expressed growth factors.

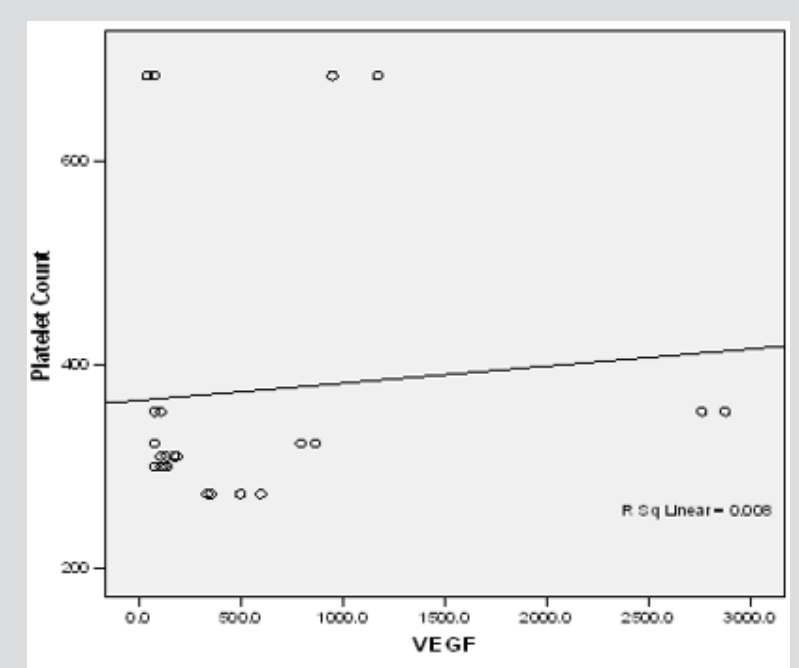

Figure 1a: Linear regression of platelet concentrate against VEGf (vascular endothelial growth factor).

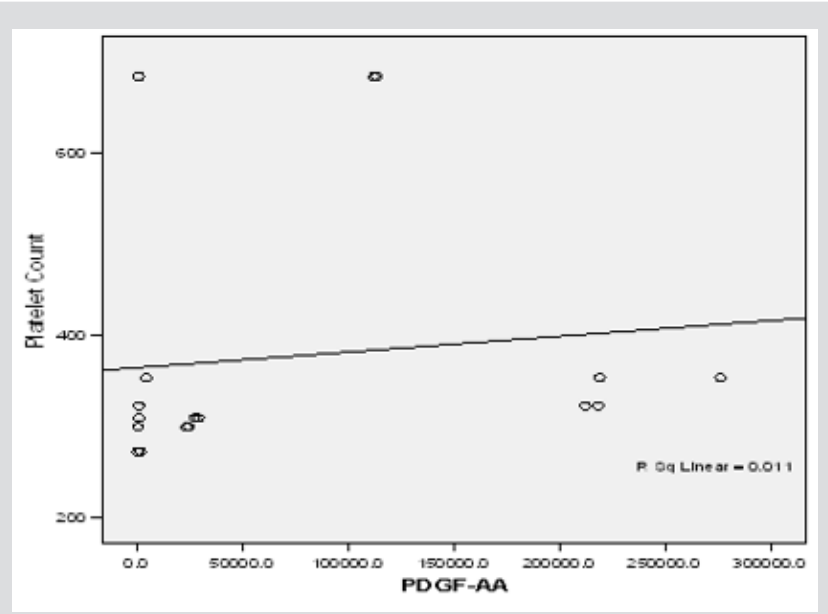

Figure 1b: Linear regression of platelet concentrate against PDGF-AA (Platelet derived growth factor -AA).

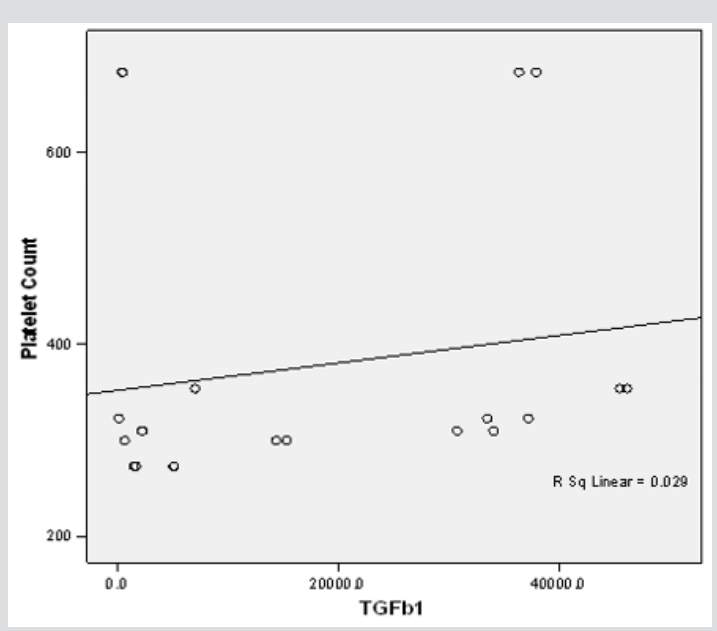

Figure 1c: Linear regression of platelet concentrate against TGF beta-1(Transforming growth factort beta-1).

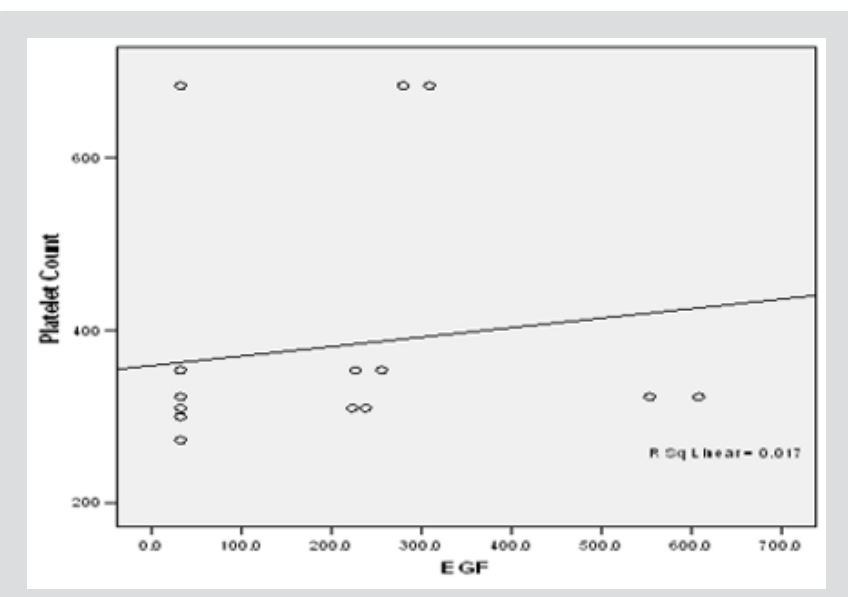

Figure 1d: Linear regression of platelet concentrate against TGF beta-1(Transforming growth factort beta-1).

\section{Comparison of Growth Factor Levels in Healthy Donor (PRP vs PPP)}

A similar trend was noticed in the GFs expression in the healthy group, but the mean value appears higher in the healthy donor. The exception is the expression of VEGF and TGF-beta1 which are higher in the diabetic group. As explained above PPP was used as the benchmark in this study. EGF in PRP to PPP was $(463 \pm 259 \mathrm{pg} / \mathrm{ml}$ : $31.9 \mathrm{pg} / \mathrm{ml})$ which is a fold increase; FGF $(136 \pm 110.6 \mathrm{pg} / \mathrm{ml}: 59.8$ $\pm 48.98 \mathrm{pg} / \mathrm{ml})$, a 2 fold increase, PDGF-AA (123.6 $\pm 8.30 \mathrm{ng} / \mathrm{ml}: 1.51$ $\pm 1.04 \mathrm{ng} / \mathrm{ml})$ a 70 fold increase, VEGF $(0.688 \pm 0.808 \mathrm{ng} / \mathrm{ml}: 0.136 \pm$ $0.132 \mathrm{ng} / \mathrm{ml})$ a 40 fold increase and IGF $(122.7 \pm 20.89 \mathrm{pg} / \mathrm{ml}: 128.4$ $\pm 21.57 \mathrm{pg} / \mathrm{ml}$ ), no increase. The activities of P-Selectin estimating level of platelet activation of PRP $(275.1 \pm 115.2 \mathrm{pg} / \mathrm{ml})$ are again two-fold higher compared with PPP $(117.9 \pm 129.8 \mathrm{pg} / \mathrm{ml})$. Table 1 showed the $n$-fold increase in the expression of the GFs in both diabetic patients and healthy volunteer as it relates to the chosen confidence interval of $\mathrm{P}=0.05$. A linear regression analysis was used to determine whether a correlation existed between platelet count and growth factor concentration in the diabetic group. The correlation was done for the four most highly expressed GFs (VEGF, 
TGF, PDGF and EGF). Figure 2a-2d is the graphic illustration of the correlation. None of the parameters was statistically significant predictor of PRP count, this might be due to the small sample size or to apparently paradoxically low secretion of GFs in patients with very high platelet concentration. Surprisingly overall, there is no evidence of a linear relationship between platelet count and GFs expression.

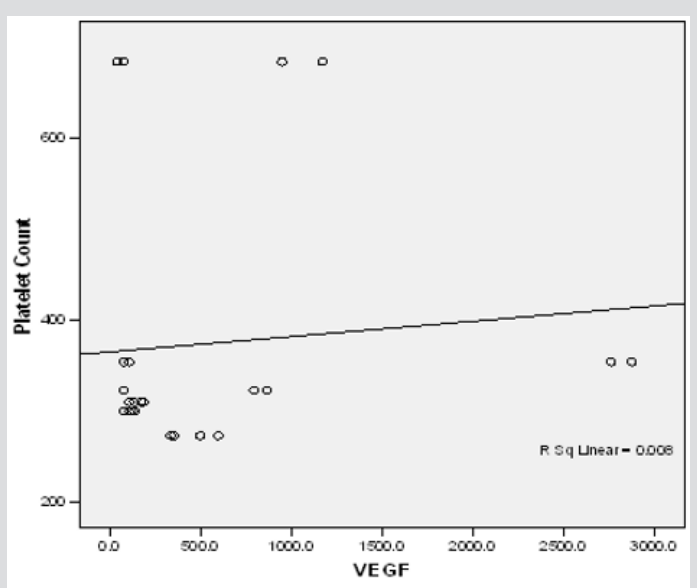

Figure 2a: Plot of Platelet concentrate against VEGF.

The 4 graphs representing linear regression of the 4 most expressed growth factors with platelet count in diabetic patients.

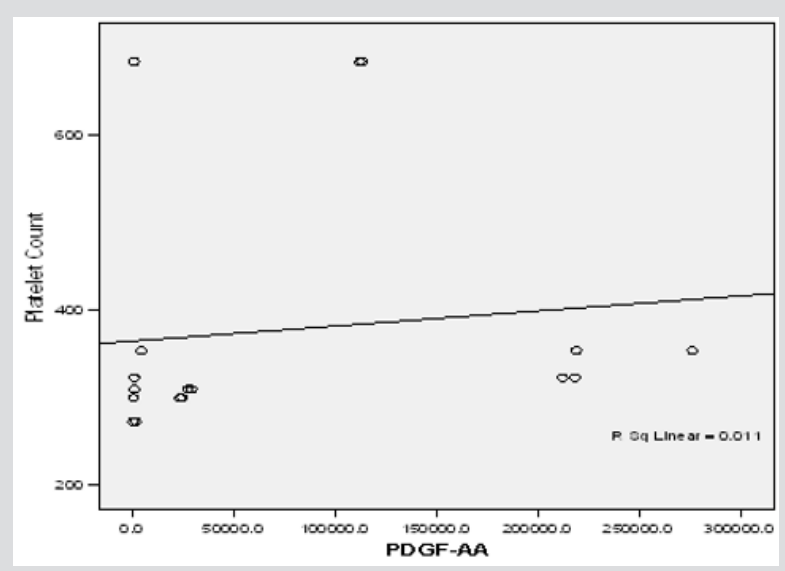

Figure 2b: Plot of Platelet concentrate against PDGF.

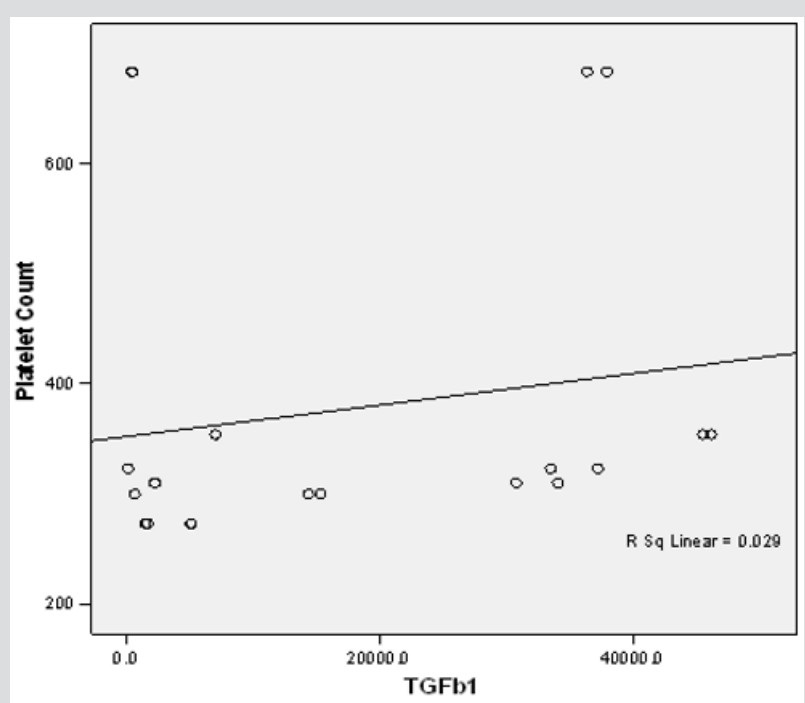

Figure 2c: Plot of Platelet concentrate against TGF-beta 1.

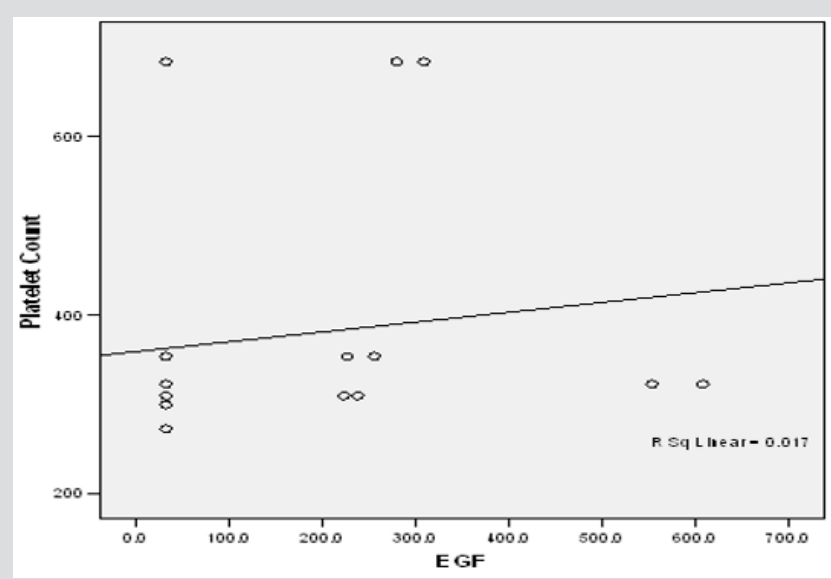

Figure 2d: Plot of Platelet concentrate against EGF.

There is no evidence of a linear relationship between platelet count and the expression growth factor (the $95 \%$ confidence interval for the slope contains the value zero and therefore is not statistically significant) the $p$ values are as follow PDGF -0.7157,VEGF-0.6616,TGF-1-0.374, EGF -0.6489.

Table 1b: The expressions of growth factors were measured in duplicate for the 14 health volunteers.

\begin{tabular}{|c|c|c|c|c|c|}
\hline $\begin{array}{c}\text { Growth factors in } \\
\text { Healthy volunteers }\end{array}$ & Comparison increase & n-fold & $\begin{array}{c}\text { Lower 95\% } \\
\text { (Confidence interval) }\end{array}$ & $\begin{array}{c}\text { Upper 95\% } \\
\text { (Confidence interval) }\end{array}$ & P- Values \\
\hline EGF & PRP vs PPP & 11.0756 & 8.3872 & 14.626 & 2.691 \\
\hline FGF-2 & PRP vs PPP & 1.9372 & 1.3944 & 100.957 & 6.0001 \\
\hline PDGF-AA & PRP vs PPP & 69.9147 & 48.4172 & 3.095 & $<.0001$ \\
\hline VEGF & PRP vs PPP & 4.4117 & 27.2235 & 1.014 & $<.0001$ \\
\hline TGFb1 & PRP vs PPP & 38.9119 & 0.9003 & 4.019 & 0.1272 \\
\hline IGF & PRP vs PPP & 0.9553 & 2.3977 & 4.0001 \\
\hline SCD62P & PRP vs PPP & 3.1759 & $<.0001$ \\
\hline
\end{tabular}

Table 1a and 1b Comparison of expression of growth factors from PRP and PPP in both Diabetic patients and Healthy Donors with their level of significance. 


\section{Discussion}

All measurements were performed in duplicate using validated commercially available ELISA and millipore kit, and coefficient of variance was $<5 \%$. For all analysis, the PRP/PPP initially generated was used stored in -800C and was then activated just before analysis of the GFs. There are published studies that have shown that deep freezing is a safe method for releasing intracellular thrombocyte GFS [13]. The Platelets count from the diabetic patient's serum and from the PRP were within acceptance range and corresponded to values previous reported in literature [14]. The correlation between the GFs and the platelet count from the PRP were weak, this might be due to small sample size, or might be due to isolated incidents of inefficient concentration of PRP. From the clinical point of view, it is important that the platelet concentration process is reliable by using a tested platelet separation device. The data obtained from this study further supports other published series, neither the direct platelet count nor the platelet in PRP could predict the desired expression of the GFs [15].

It is crucial to minimize platelet activation that occurs during PRP preparation, this is because activation results in untimely release of GFS, which could result in deletion of expressed GFs at the point of use. Furthermore, ability to store- frozen PRP has clinical implications from treating patients that might require repeated application of activated PRP in other to achieve the desire end point such as wound healing. In our study P-selectin was measured after activation of PRP as means of defining the degree of activation, and a 3-fold increase was noticed when PRP was compared with PPP in the healthy donor and the diabetic patient. In a similar study by Eppley et al. P-selectin was measured after centrifugation of PRP before the activation and they reported that there was no significant change in the level as compared with the whole blood [1]. Although our approach was different to the Eppley et al. the levels of GFs expression in our study compares favorably to other published series $[4,15]$.

We compared the GF expression profile between PRP and PPP, contrary to the popular believe that PPP has no GF expression and only useful as a sealant [8], we noticed a significant expression of some growth factors from PPP, particularly from PDGF and TGF were the most prominent amongst the other expressed GF. Theoretically, levels of platelet-derived growth factors in PRP might be expected to depend on the number of platelets involved, which have previously been reported in some studies [15]. However, from our study we were not able to demonstrate a linear relationship. Eppley et al., concluded that the very best correlation was seen with TGF-beta1, which was still quite low, he suggested little correlation between platelet number and GF concentration and that content of the platelets varies from patient to patient. He further explained that this result might be due to by high individual variability in cellular production or storage of cytokines [5]. Of all the GFs identified in the wound cascade, the concentration of IGF-1 was not significantly increased. IGF-1 is primarily excreted by the liver into the blood plasma; hence the value of IGF is marginally higher in PRP than PPP in both groups. The findings in our study correlate with previous reports $[1,6,15,16]$, which emphasized significant interpatient variability of growth factors expression.

The method of PRP preparation in our study achieved a significant level of GFs expression but there are practical drawbacks to this technique, limitation such as longer time of preparation and problem of fixed volume of whole blood needed to operate the device. However, it is debatable whether this is the most effective method, but it's clinically convenient and user-friendly for the preparation process. In our study, there is an age and gender mismatch between our healthy volunteer and the diabetic group, however, other studies have also suggested that gender and age are not predictive factors in determining GFs expressions [16]. Further study with a control disease group will be more helpful to address the effect of chronic disease on GFs expression. Reliable prediction of GFs levels in PRP samples is necessary to ensure reliable and reproducible use of PRP for clinical treatment, since the regenerative potency of PRP undoubtedly depends on its GFs Level [17]. It is also therefore clinically relevant to recognize that each PRP processing method may differ as regards to platelet counts and activation techniques which may have implications for GF release and expressions [16].

Autologous derived Platelet rich plasma is promising novel technique that has found various applications beyond its initial application in periodontics and maxillofacial surgery [1,11,14]. It has been used in sport medicine with positive results in areas of cruciate ligament reconstruction, soft tissue injury, tendon and ligament injury. PRP have also been applied bariatric surgery, Brady et al. [18]. used PRP via an endoscopic delivery system, after laparoscopic Roux-en Y gastric bypass procedure to prevent bleeding and infection anastomotic leaks. He concluded that the use of PRP contributes significantly to wound healing and improved clinical outcome Furthermore, autologous fibrin derived from PPP, has been used as a biological sealant during aortic anastomosis during thoracoabdominal aortic surgery.

In a randomized control trial of endovascular repair of abdominal aortic aneurysm repair, with 50 patients in each arms of the treatment group, Saratzis et. al, reported a significantly lower postoperative hospital stay and lower inguinal wound-related complication [19]. Following clinical trials on the efficacy of PDGFBB (Becaplermin) in treating neuropathic diabetic foot ulcers, evidence suggests that bests results are obtained with PDGF-BB in combination with the aggressive debridement, a process which removes senescent fibroblast and pathogens from chronic wounds $[4,20,21]$. It was postulated that autologous derived Platelet-rich plasma has an advantage over the single recombinant GF, because the GFs have synergistic effect of the GF on each other and they promote mitogenesis of mesenchymal stem cells at the wound site [22]. The postulated therapeutic advantage has not been proven in any clinical trial. 


\section{Conclusion}

Results from this study demonstrated that platelets can be sequestered and concentrated 6-fold from whole blood. Furthermore, PRP (either from diabetic patients or healthy volunteers) contains significantly higher levels of GFs expression compared with PPP, which has potential benefits on wound healing. Furthermore, we demonstrated that activating PPP will release similar expression of growth factors as seen in PRP but to a lesser degree. The prediction of GFs expression based on platelet counts have not been possible in this study. Further investigation might be needed to resolve the issues of unpredictability of growth factor expression from PRP. Furthermore, a technique whereby rapid quantification of GFs at the bedside may hold therapeutic potential.

\section{References}

1. Eppley BL, Woodell JE, HIggins J (2004) Platelet quantification and growth factor analysis from platelet-rich plasma: implications for wound healing. Plast Reconstr Surg 114(6): 1502-1508.

2. Margolis DJ, Kantor J, Santanna J, Strom BL, Berlin JA (2001) Effectiveness of platelet releasate for the treatment of diabetic neuropathic foot ulcers. Diabetes care 24(3): 483-488.

3. Everts PA, Overdevest EP, Jakimowicz JJ, Oosterbos JP, Schonberger JP, et al. (2007) The use of autologous platelet-leukocyte gels to enhance the healing process in surgery, a review. Surg Endosc 21(11): 2063-2068.

4. Alsousou J, Thompson M, Hulley P, Noble A, Willett K (2009) The Biology of platelet-rich plasma and its application in trauma and orthopedic surgery. J Bone Joint Surg Br 91(8): 987-996.

5. Marx RE, Carlson ER, Eichstaedt RM, Schimmele SR, Strauss JE, et al (1998) Platelet-rich plasma: Growth factor enhancement for bone grafts. J Oral Maxillofacial Surg 85(6): 638-646.

6. Kawase T (2015) Platelet-rich plasma and its derivatives as promising bioactive materials for regenerative medicine: basic principles and concepts underlying recent advances. Odontology. 103(2): 126-135.

7. Anitua E (2001) The use of plasma-rich growth factors (PRGF) in oral surgery. Pract Proced Aesthet Dent 13(6): 487-493.

8. Borzini P, Mazzucco L (2007) Platelet-rich plasma (PRP) and platelet derivatives for topical therapy. What is true from the biologic viewpoints? ISBT Sci Ser 2(1): 272-281.

9. Tabata Y (2003) Tissue regeneration based on growth factor release. Tissue Eng 9(Suppl): 5-15.

\section{ISSN: 2574-1241}

\section{DOI: 10.26717/BJSTR.2019.18.003191}

Akinfemi A Akingboye. Biomed J Sci \& Tech Res

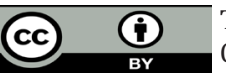

This work is licensed under Creative

Commons Attribution 4.0 License

Submission Link: https://biomedres.us/submit-manuscript.php
10. Werner S, Grose R (2003) Regulation of wound healing by growth factor by growth factors and cytokines. Physiol Rev 83: 835-870.

11. Weibrich G, Kleis WKG, Hafner G (2002) Growth factor levels in the platelet-rich plasma produced by 2 different methods: curasan-type kit versus PCCs PRP system. Int J Oral Maxillofac Imp 17(2): 184-190.

12. Choukroun J (2014) Advanced PRF, \&i-PRF: platelet concentrates or blood concentrates? J Periodont Med Clin Practice 1: 3.

13. Saratzis N, Saratzis A, Melas N, Kiskinis D (2008) Non- activated autologous platelet-rich plasma for the prevention of inguinal woundrelated complications after endovascular repair of abdominal aortic aneurysms. J Extra Corpo Technol 40(1): 52-56.

14. Sekido Y, Morishima Y, Ohya K (1987) Activity of platelet- derived growth factor (PDGF) in platelet concentrates and cryo- preserved platelets determined by PDGF bioassay. Vox Sang 52(1-2): 27-30.

15. Weibrich G, Kleis WKG, Hafner G, Hitzler WE (2002) Growth factor levels in platelet-rich plasma and correlations with donor age, sex and platelet count. J Cranio Max Fac Surg 30(2): 97-102.

16. Kevy SV, Jacobson MS, Fagnant A (2001) Preparation of growth factor enriched autologous platelet gel. Presented at the $27^{\text {th }}$ Annual Meeting of the Society for Biomaterial, St. Paul, Minn April 2001.

17. Kevy, SV, Jacobson MS (2004) Comparison of the methods for point of care preparation autologous platelet gel. J Extra Corpor technol 36(1): 28-35.

18. Zhu SJ, Choi BH, Jung JH, Lee SH, Huh JY, et al. (2006) A comparative histologic analysis of tissue-engineered bone using platelet-rich plasma and platelet-enriched fibrin glue. Oral Surg Oral Med Oral Pathol Oral Radiol Endod 102(2): 175-179.

19. Tawes RL Sydorak GR, DU Vall TB (1994) Autologous fibrin glue: the last step on operative hemostasis. Am J Surg 168(2): 120-122.

20. Steed DL (2006) Clinical evaluation of recombinant human plateletderived growth factor for the treatment of lower extremity ulcer. Plast. Reconstr Surg 117(Suppl): 143S-151S.

21. Steed DL, Donohoe D, webster MW, Lindsley L (1996) Effect of extensive debridement and treatment on the healing rate of diabetic foot ulcer. Diabetic Ulcer group. J Am Coll Surg 183(1): 61-64.

22. Goldman R (2004) Growth factors and chronic wound healing: Past, Present and Future. Adv skin wound care 17(1): 24-35.

23. Brady C, Vang S, Christensen K, Jack Isler, David Holt (2006) Use of platelet gel in bariatric surgery. J Extracorp Technol 38(2): 161-164.

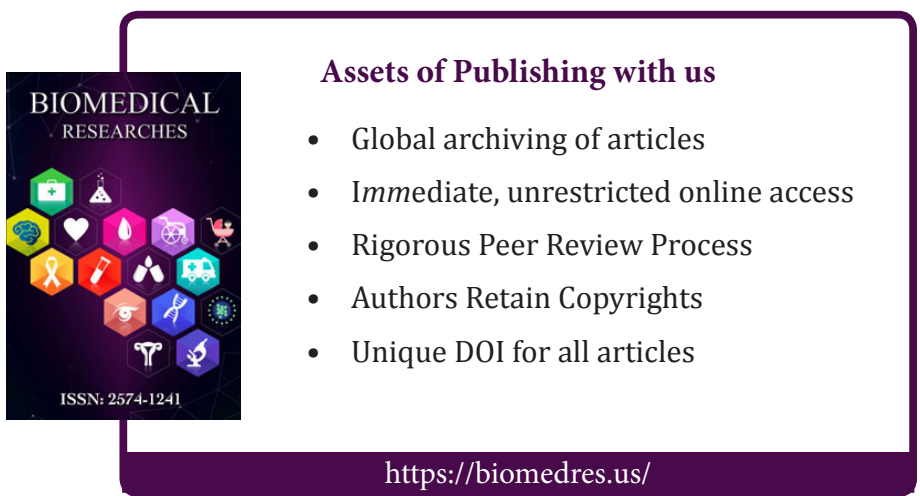

